\title{
Gestantes com câncer de mama em tratamento quimioterápico com doxorrubicina, ciclofosfamida e docetaxel em hospital oncológico de referência: um relato de dois
}

\section{casos}

Pregnant women with breast cancer under chemotherapy treatment with doxorubicin, cyclophosphamide and docetaxel at a reference oncology hospital: two cases report

Mujeres embarazadas con cáncer de mama en tratamiento con quimioterapia con doxorrubicina, ciclofosfamida y docetaxel en un hospital de oncología de referencia: un relato de dos casos

Recebido: 30/11/2021 | Revisado: 04/12/2021 | Aceito: 10/12/2021 | Publicado: 1812/2021

Paula Nogueira da Silva

ORCID: https://orcid.org/0000-0002-7321-1365 Grupo Oncoclínicas, Brasil

E-mail: pnogueira.silva5@gmail.com

Patrícia Marques Soares Valente ORCID: https//orcid.org/0000-0002-6339-2385

Universidade Federal Fluminense, Brasil E-mail: pvalente@id.uff.br

Selma Rodrigues de Castilho

ORCID: https://orcid.org/0000-0003-0272-4777

Universidade Federal Fluminense, Brasil E-mail: selmarc@id.uff.br

Juliana de Oliveira Miguel

ORCID: https//orcid.org/0000-0002-6339-2385

Instituto Nacional do Câncer, Brasil

E-mail: dom.juliana@yahoo.com.br

Maria Fernanda Barbosa

ORCID: https://orcid.org/0000-0002-2018-6151

Instituto Nacional do Câncer, Brasil

E-mail: barbosa.mf@gmail.com

Flavia Campos Barcelos

ORCID: https://orcid.org/0000-0001-6193-6468

Instituto Nacional do Câncer, Brasil

E-mail: fbarcelos1979@gmail.com

\begin{abstract}
Resumo
O objetivo deste relato de dois casos de câncer de mama gestacional (CMG) é contribuir com a lacuna empírico teórica sobre as repercussões para o binômio mãe-filho, resultantes da associação do câncer na gestação. Foram recrutadas para o acompanhamento farmacoterapêutico duas pacientes com câncer de mama em terapia com doxorrubicina, ciclofosfamida e docetaxel. Paciente 1, 42 anos, grávida de 26 semanas, parda, gari, mãe de seis filhos, diagnosticada com câncer em mama esquerda do tipo carcinoma ductal invasivo (CDI), estadiamento III-B, grau III, receptor hormonal (RH) negativo, HER-2 positiva, apresentando hipertensão arterial, tabagista há 20 anos com fumo de rolo, ex-etilista. Apresentou reações adversas a medicamentos (RAM) durante a quimioterapia (QT): constipação; fadiga; hiperpigmentação; mucosite e anemia; hiperglicemia e aumento da fosfatase alcalina. Paciente 2, 39 anos, grávida de 30 semanas, desempregada, mãe de três filhos, diagnosticada com câncer em mama direita do tipo CDI, estadiamento III-A, grau II, RH negativo, HER-2 negativo, triplo negativa, apresenta histórico de Púrpura trombocitopênica imune (PTI) e sem histórico social de tabagismo ou etilismo. Apresentou RAM durante a QT: boca seca; fadiga; alteração de paladar; diarreia; hiperpigmentação; anemia; hiperglicemia; aumento da fosfatase alcalina e de transaminase oxalacética (TGO). O CMG é considerado um evento desafiador e raro, apesar da incidência com o retardo da gravidez para após os 30 anos, estar aumentando. Além disso, é importante a participação ativa da equipe multiprofissional centrada no bem-estar materno-fetal. Neste estudo as principais RAM apresentadas pelas pacientes foram leves, principalmente gastrointestinais e bioquímicas.

Palavras-chave: Gravidez; Neoplasias da mama; Antineoplásicos; Efeitos colaterais e reações adversas relacionados a medicamentos.
\end{abstract}




\begin{abstract}
The objective of this report of two cases of pregnancy-associated breast cancer (PBC) is to contribute to the theoretical empirical gap about the repercussions for the mother-child binomial, resulting from the association of cancer during pregnancy. Two breast cancer patients receiving doxorubicin, cyclophosphamide and docetaxel therapy were recruited for pharmacotherapeutic follow-up. Patient 1, 42 years old, 26 weeks pregnant, mixed race, street sweeper, mother of six, diagnosed with left breast cancer of the invasive ductal carcinoma (IDC), stage III-B, grade III, hormone receptor (RH) negative, HER-2 positive, with high blood pressure, smoker for 20 years with tobacconist, former alcoholic. Presented adverse drug reactions (ADR) during chemotherapy (CT): constipation; fatigue; hyperpigmentation; mucositis and anemia; hyperglycemia and increased alkaline phosphatase. Patient 2, 39 years old, 30 weeks pregnant, unemployed, mother of three, diagnosed with ICD-type right breast cancer, stage III-A, grade II, RH negative, HER-2 negative, triple negative, has a history of Immune thrombocytopenic purpura (ITP) and no social history of smoking or drinking. Presented ADR during QT: dry mouth; fatigue; change of taste; diarrhea; hyperpigmentation; anemia; hyperglycemia; increase in alkaline phosphatase and oxaloacetic transaminase (GOT). $\mathrm{PBC}$ is considered a challenging and rare event, although the incidence with delayed pregnancy beyond the age of 30 is increasing. In addition, the active participation of the multidisciplinary team focused on maternal-fetal well-being is important. In this study, the main ADR presented by the patients were mild, mainly gastrointestinal and biochemical.
\end{abstract}

Keywords: Pregnancy; Breast cancer; Antineoplastics; Drug-related side effects and adverse reactions.

\title{
Resumen
}

El objetivo de este reporte de dos casos de cáncer de mama gestacional (CMG) es contribuir al vacío empírico teórico sobre las repercusiones para el binomio madre-hijo, resultante de la asociación del cáncer durante el embarazo. Se reclutó a dos pacientes con cáncer de mama que recibían terapia con doxorrubicina, ciclofosfamida y docetaxel para seguimiento farmacoterapéutico. Paciente 1, 42 años, 26 semanas de embarazo, mestiza, limpiadora de calles, madre de seis hijos, diagnosticada con cáncer de mama izquierdo del tipo carcinoma ductal invasivo (CDI), estadio III-B, grado III, receptor hormonal (RH) negativo, HER-2 positivo, hipertenso, fumador desde hace 20 años con estanco, ex alcohólico. Reacciones adversas a medicamentos (RAM) presentadas durante la quimioterapia (QT): estreñimiento; fatiga; hiperpigmentación; mucositis y anemia; hiperglucemia y aumento de la fosfatasa alcalina. Paciente 2, 39 años, 30 semanas de embarazo, desempleada, madre de tres hijos, diagnosticada con cáncer de mama derecho tipo ICD, estadio III-A, grado II, RH negativo, HER-2 negativo, triple negativo, tiene antecedentes de inmunodeficiencia humana. púrpura trombocitopénica (PTI) y sin antecedentes sociales de fumar o beber. RAM presentado durante QT: sequedad de boca; fatiga; cambio de gusto; diarrea; hiperpigmentación; anemia; hiperglucemia; aumento de fosfatasa alcalina y transaminasa oxaloacética (TGO). La CMG se considera un evento difícil y poco común, aunque la incidencia de embarazo tardío después de los 30 años está aumentando. Además, es importante la participación activa del equipo multidisciplinario enfocado en el bienestar materno-fetal. En este estudio, las principales RAM que presentaron los pacientes fueron leves, principalmente gastrointestinales y bioquímicas.

Palabras clave: Embarazo; Neoplasias de la mama; Antineoplásicos; Efectos colaterales y reacciones adversas relacionados con medicamentos.

\section{Introdução}

De acordo com o Instituto Nacional de Câncer José de Alencar (INCA), o câncer de mama é considerado uma neoplasia de origem multifatorial, sendo a heterogeneidade tumoral um dos maiores desafios para os desfechos positivos do seu tratamento (Brasil, 2020). Segundo estimativas da Organização Mundial de Saúde (OMS) - Globocan 2018, dentre os diversos tipos de câncer, o de mama se destacou por ter sido o mais incidente $(24,2 \%)$ e a primeira causa de morte por câncer (15\%) entre a população feminina mundial (Ferlay et al., 2018).

O câncer de mama gestacional (CMG) é definido como o câncer de mama diagnosticado três meses antes do aborto, durante a gravidez ou um ano após o parto. Os tipos de câncer mais prevalentes associados com a gestação são: melanoma, mama, colo do útero, linfomas e leucemias, sendo o de mama, o mais comum na gestação, representando $25 \%$ de todas as neoplasias malignas apresentadas, entretanto ainda é considerado um evento raro (Case, 2016; Kettelhut \& Modena, 2008; Prado et al., 2020).

Dentre os fatores de risco para o desenvolvimento do câncer de mama, destacam-se: sexo feminino, idade, histórico familiar, menopausa tardia (após 50 anos de idade), obesidade, tabagismo, ingestão de álcool. Além disso, a nuliparidade e a gestação após os 30 anos de idade também são considerados fatores de risco (Bell et al., 2013). 
A incidência de CMG tem tendência crescente com a modernidade devido principalmente à nova realidade feminina, acarretando na decisão de postergar a maternidade. A incidência é de aproximadamente 15 a 35 por 100.000 partos, com mais casos diagnosticados durante o primeiro ano pós-parto, o risco aumenta com o avançar da idade, com maior incidência em mulheres de 32 a 38 anos (Case, 2016). A gravidez apresenta efeito protetor para o desenvolvimento do câncer de mama associado à idade precoce de primeira gestação e à paridade. Estudos afirmam que a primeira gravidez antes dos 30 anos reduz o risco em 25\% em comparação com as nulíparas (Bell et al., 2013; Merviel et al., 2011; Monteiro et al., 2019).

O CMG geralmente é diagnosticado em fase avançada, apresenta tipo histológico pouco diferenciado, normalmente o carcinoma ductal invasivo (CDI) e pior prognóstico, geralmente com receptores hormonais negativos. O atraso no diagnóstico é reportado em 40\% dos casos de CMG. O diagnóstico retardado está associado principalmente às alterações fisiológicas da mama na gravidez, dificultando o exame clínico e a detecção de pequenas massas, interpretação da mamografia e ultrassonografia mamária, podendo confundir os profissionais de saúde. Outro fator é a hesitação das gestantes em realizar a ultrassonografia por medo de dano fetal. Evitar o atraso no diagnóstico é vital, visto que o atraso de um mês no diagnóstico pode aumentar o risco de envolvimento nodal em até 2\% (Case, 2016; Kettelhut \& Modena, 2008; Monteiro et al., 2019; Silva et al., 2018).

O tratamento de mulheres com CMG apresenta um cenário único e desafiador, sendo necessário o equilíbrio entre o risco-benefício tanto para a mãe quanto para o feto. O acompanhamento dessas pacientes deve ser realizado por uma equipe multiprofissional e a presença do farmacêutico nesta equipe visa conferir mais segurança e esclarecer as dúvidas da equipe quanto a prescrição de medicamentos álgicos e de suporte apropriados para a gravidez, minimizando assim os riscos para o feto e possíveis impactos na gestação. (Avery \& Williams, 2015; Case, 2016; Cordeiro \& Gemignani, 2017; Suzuki et al., 2019).

A gestante com suspeita de câncer de mama deve ser submetida aos exames de diagnóstico, como a mamografia com proteção abdominal, ultrassonografia da mama e biópsia por punção aspirativa de agulha fina (PAFF) (Bell et al., 2013).

Em relação aos tipos de tratamento para o CMG destacam-se a cirurgia e a quimioterapia. A radioterapia é contra indicada, visto que o feto é submetido a alto risco de toxicidade (Prado et al., 2020). A cirurgia é considerada o tratamento de primeira linha, podendo ser realizada com segurança em qualquer estágio da gravidez, com complicações mínimas na maioria dos casos (Silva et al., 2018). A quimioterapia deve ser evitada no primeiro trimestre, no qual ocorre a organogênese, com maior risco de ocorrência de aborto espontâneo e teratogênese fetal em até $20 \%$ dos casos. A partir do segundo trimestre, o risco de malformação fetal se reduz para 3\%, porém ainda pode resultar em prematuridade e baixo peso ao nascer (Case, 2016; Cipriano \& Oliveira, 2016; Cordeiro \& Gemignani, 2017; Kettelhut \& Modena, 2008; Prado et al., 2020).

Os antineoplásicos mais comumente utilizados e considerados relativamente seguros para o tratamento do CMG são doxorrubicina, ciclofosfamida, fluorouracila e taxanos (Case, 2016; Kettelhut \& Modena, 2008). Alguns estudos indicam que a quimioterapia deve ser interrompida após a $35^{\mathrm{a}}$ semana de gestação, visando reduzir os riscos de neutropenia, mielossupressão fetal, hemorragia e infecção materna. O aleitamento materno é contraindicado devido à excreção dos antineoplásicos no leite (Cipriano \& Oliveira, 2016; Prado et al., 2020; Silva et al., 2018).

O protocolo AC-T (doxorrubicina associada a ciclofosfamida seguido de paclitaxel ou docetaxel) é comumente utilizado pelas pacientes com CMG e está associado a diversas reações adversas a medicamentos (RAM), podendo impactar no cotidiano das pacientes. Destaca-se, o alto perfil emetogênico, constipação, alopecia e cardiotoxicidade, hipersensibilidade, hematotoxicidade, principalmente neutropenia febril (15-20\%), mialgia, artralgia e neuropatia periférica (Biganzoli et al., 2016; Castro et al., 2013; Chiu et al., 2017).

Há falta de estudos prospectivos relacionados ao CMG, desta forma, apesar de estudos internacionais não demonstrarem diferença significativa nas taxas de sobrevida entre pacientes com CMG e não CMG, sugere-se que o 
prognóstico é pior em pacientes com CMG (Kettelhut \& Modena, 2008). Nesse contexto, esse relato de dois casos de CMG tem por objetivo contribuir com a lacuna empírico teórica sobre as repercussões para o binômio mãe-filho, resultantes da associação do câncer na gestação.

\section{Metodologia}

Este artigo é um relato de dois casos, quantitativo e descritivo (Pereira et al, 2018). Inicialmente, os dados foram coletados de prontuários clínicos. Em seguida, as pacientes foram entrevistadas pelo farmacêutico responsável pelo serviço de farmácia clínica para complementar as informações. O estudo foi realizado em um hospital de referência em oncologia do estado do Rio de Janeiro. O projeto foi aprovado no Comitê de Ética em Pesquisa (CEP) do Hospital federal oncológico, sob o CAAE: 10419319.9.0000.5274 e envolveu duas gestantes, no segundo trimestre de gravidez, diagnosticadas com câncer de mama em tratamento neoadjuvante com os medicamentos: doxorrubicina e ciclofosfamida seguidos de docetaxel (AC-T) em um hospital de referência em oncologia do Rio de Janeiro.

\section{Relato de Casos}

Paciente 1: 42 anos, grávida de 26 semanas, parda, gari, mãe de seis filhos, diagnosticada com câncer em mama esquerda do tipo CDI no exame histopatológico em 02/2019, estadiamento III-B, grau III, receptor hormonal (RH) negativo, HER-2 positiva, apresentando hipertensão arterial, hipertireoidismo e Índice de Massa Corpórea (IMC) 24,5, classificado como normal de acordo com o Ministério da Saúde, tabagista há 20 anos com fumo de rolo, ex-etilista. Apresentou histórico familiar de câncer de estômago (avó materna) e câncer de pulmão (avô paterno). Relatou, em consulta farmacêutica, as seguintes RAM durante o ciclo de AC: constipação G1; fadiga G1; hiperpigmentação G1; mucosite G1 (Tabela 1). Os achados dos exames laboratoriais indicaram: anemia G1; hiperglicemia e aumento da fosfatase alcalina (Tabela 1). Devido à anemia após primeiro ciclo de AC, a paciente foi submetida a hemotransfusão antes dos ciclos de quimioterapia subsequentes. Realizou apenas os quatro primeiros ciclos do protocolo AC, devido à progressão nodal e local, sendo necessária a troca de protocolo clínico para tratamento paliativo com gencitabina associada à cisplatina (GemCis). Em 08/2019 a paciente foi internada para realização do parto, com 37 semanas. Anormalidades fetais não foram reportadas. Após o parto, em 08/19, a paciente foi submetida ao primeiro ciclo de GemCis, apresentando neutropenia febril G3, pancitopenia G1 e derrame pleural, sendo submetida à internação hospitalar. Em 09/2019, apresentou dispneia intensa sendo submetida a nova internação hospitalar. Paciente foi a óbito, devido à evolução da neoplasia, em 09/2019.

Paciente 2: 39 anos, grávida de 30 semanas, desempregada, mãe de três filhos, diagnosticada com câncer em mama direita do tipo CDI no exame histopatológico, em 03/2019, estadiamento III-A, grau II, RH negativo, HER-2 negativo, triplo negativa, apresentou histórico de Púrpura trombocitopênica imune (PTI), IMC 25,5 classificado como sobrepeso e sem histórico social de tabagismo ou etilismo. Apresentou histórico familiar de câncer de mama (tia materna). Relatou em consulta farmacêutica as seguintes RAM durante o ciclo de AC: boca seca G1; fadiga G1; hiperpigmentação G1 (Tabela 1). Os achados dos exames laboratoriais indicaram: anemia G1; hiperglicemia; aumento da fosfatase alcalina e de transaminase oxalacética (TGO). Durante os ciclos de docetaxel relatou: alteração do paladar G1 e diarreia G1, em relação aos exames laboratoriais apresentou anemia G1 (Tabela 1). Em 07/2019 a paciente foi internada para realização do parto com 40 semanas. Anormalidades fetais não foram reportadas. Realizou o protocolo AC-T, seguido de mastectomia radical. Devido à progressão pulmonar, iniciou protocolo paliativo com ciclofosfamida, metrotexato associado a fluorouracila (CMF). Após o primeiro ciclo de CMF a paciente foi internada devido a flair PIT (plaquetopenia). Em 09/2020 internação hospitalar devido a dispneia, astenia e dor. Paciente foi a óbito devido à progressão de doença em 09/2020. 
Tabela 1:Reações adversas G1/G2 do protocolo ACT por número de paciente.

\begin{tabular}{|cll}
\hline Desordens por sistema (\%) & \multicolumn{1}{c}{ Reações adversas } & n \\
\hline \multirow{2}{*}{ Gastrointestinais (28) } & Alteração de paladar & 1 \\
& Boca seca & 1 \\
& Constipação & 1 \\
& Diarreia & 1 \\
& Mucosite & 1 \\
& Total & $\mathbf{5}$ \\
\hline \multirow{2}{*}{ Dermatológicas (22) } & Alopecia & 2 \\
& Hiperpigmentação & 2 \\
& Total & 4 \\
\hline \multirow{2}{*}{ Músculo-esqueléticas (11) } & Fadiga & $\mathbf{2}$ \\
& Total & $\mathbf{2}$ \\
\hline \multirow{2}{*}{ Bioquímicas (39) } & Anemia & $\mathbf{2}$ \\
& Glicemia $\geq 100$ mg/dl) & 2 \\
& Plaquetopenia & 2 \\
& Elevação de transaminases & 1 \\
& Total & 7 \\
\hline Total (100) & & 18 \\
\hline
\end{tabular}

Fonte: Autores.

\section{Discussão}

Um dos fatores de risco para o desenvolvimento do câncer de mama é a nuliparidade ou a primeira gestação após os 30 anos, fator cada vez mais observado devido à modernidade. Bell et al. (2013) estudaram mulheres com câncer de mama, comparando-as com mulheres do grupo não $\mathrm{CMG}$, e reportaram que mulheres com primeira gravidez após os 35 anos apresentam maior risco de desenvolver CM na vida do que nulíparas (Kettelhut \& Modena, 2008; Monteiro et al., 2019). Ambas as pacientes acompanhadas neste estudo tiveram gestações antes de 35 anos.

As pacientes do estudo apresentaram histórico familiar de câncer de estômago, pulmão e de câncer de mama de familiares de primeiro grau, neoplasias que apresentam características de hereditariedade. O histórico familiar é um fator de risco para o desenvolvimento do câncer de mama sendo indicada a realização do aconselhamento genético. A presença de histórico familiar de câncer de mama aumenta o risco em 80\% em familiares de primeiro grau (Case, 2016; Monteiro et al., 2019).

Prado et al. (2020), reportaram a faixa etária dos casos de CMG de 32 a 38 anos, Cieto et al. (2021), 27 a 44 anos, enquanto Kettelhut e Modena (2008) observaram média de idade ao diagnóstico de CMG de 33 anos. Neste estudo, as pacientes tinham idade 39 e 42 anos, idade mais avançada quando comparado aos dados de Prado et al. (2020) e Kettelhut e Modena (2008). Estudos indicam que a idade gestacional média no início da quimioterapia foi de 20 semanas (17 e 33 ), já neste estudo a faixa de idade gestacional ao diagnóstico foi de 25-30 semanas, apresentando similaridade com os dados apresentados pela literatura (Cieto et al., 2021; Cipriano \& Oliveira, 2016; Cordeiro \& Gemignani, 2017; Kettelhut \& Modena, 2008; Prado et al., 2020).

Estudos recentes sobre CMG têm demonstrado que a maioria das pacientes apresenta carcinoma do tipo CDI, expressão negativa do receptor hormonal (progesterona e estrogênio) na faixa de 49-84\% e frequentemente apresentam superexpressão do receptor 2 do fator de crescimento epidérmico humano (HER2) na faixa de 28-58\%, sendo este fenótipo associado a um perfil mais invasivo. Isto sugere que as características biológicas do tumor CMG ou de seu microambiente 
devido à gravidez podem estar acarretando em um possível aumento do risco de pior prognóstico, além do diagnóstico tardio e estadiamentos mais avançados que podem estar associados também à dificuldade da gestante em diferenciar os sintomas da gravidez com um possível nódulo. Este pior prognóstico pode apresentar impacto na sobrevida das pacientes com CMG, pacientes de mesma idade não gestantes em geral apresentam sobrevida de 80\% em cinco anos, já as com CMG apresentam 23\% a 52\% resultado significativamente menor (Bae et al., 2018; Bezerra et al., 2019; Cordeiro \& Gemignani, 2017; Kettelhut \& Modena, 2008; Souza et al., 2015).

Monteiro et al. (2019), reportaram que o estadiamento do câncer de mama entre as gestantes teve como resultado três (15\%) pacientes em IIB e 18 (85\%) pacientes nos estágios III e IV e o CDI foi o tipo histológico predominante em 20 (95\%) dos casos. Estes achados são semelhantes aos deste estudo, no qual as gestantes obtiveram diagnóstico de câncer de mama com estadiamento tardio III, tipo histológico foi o CDI e receptor hormonal negativo, entretanto uma das pacientes não apresentou superexpressão de HER2 como comumente reportado pela literatura (Bae et al., 2018; Cordeiro \& Gemignani, 2017; Kettelhut \& Modena, 2008; Monteiro et al., 2019).

Neste estudo as duas pacientes receberam tratamento neoadjuvante devido ao estadiamento avançado da doença e o tamanho do tumor. Cieto et al. (2021), relataram que das 10 gestantes em seu estudo que realizaram algum tratamento quimioterápico, $30 \%$ receberam quimioterapia neoadjuvante.

A maioria dos estudos relata que as toxicidades apresentadas pelas pacientes com CMG expostas à quimioterapia foram precoces e reversíveis incluindo: anemia, neutropenia e alopecia (Case, 2016; Cieto et al., 2021). No estudo de Cieto et al. (2021), foi evidenciado que aproximadamente $74 \%$ das gestantes apresentaram alguma complicação, durante a gravidez, sendo a anemia materna a mais frequente (43\%), neste estudo apenas uma das pacientes apresentou anemia, sendo necessário realizar transfusão sanguínea antes dos ciclos de quimioterapia, ambas apresentaram alopecia, fadiga e hiperpigmentação, sendo evidenciado principalmente RAM bioquímicas (39\%) e gastrointestinais (28\%).

Uma das complicações mais significativas relacionadas à exposição das pacientes com CMG à quimioterapia foi a neutropenia febril (Cordeiro \& Gemignani, 2017). Entretanto, no presente, estudo apenas uma paciente apresentou neutropenia febril, após o parto, durante a exposição ao novo protocolo quimioterápico paliativo, já no estudo de Walker et al. (2011) foi reportado que 4 (5\%) das 82 pacientes expostas ao protocolo AC-T não grávidas apresentaram neutropenia febril.

Nos resultados observados, nenhuma criança apresentou RAM ou complicações no parto e nem após o nascimento até o momento de conclusão do estudo, dado semelhante ao de Cieto et al. (2021), que reportaram também que os bebês nasceram com idade gestacional mediana de 37 semanas e nenhuma anormalidade fetal relatada. Outro estudo evidenciou que das 57 crianças nascidas após a gestante com CMG ser exposta à quimioterapia, $10 \%$ apresentaram complicações no parto como dificuldade respiratória, uma desenvolveu hemorragia subaracnóidea, uma nasceu com Síndrome de Down e duas com malformação congênita (Cipriano \& Oliveira, 2016).

Os locais de metástase evidenciados neste estudo foram nodal, local e pulmonar, sendo que os locais mais comuns de ocorrência de metástases relatados na literatura incluem pulmões, fígado e ossos. A fosfatase alcalina aumenta durante a gravidez devido à produção da placenta e não é indicativa de doença óssea (Case, 2016).

A diferença na mortalidade entre CMG e não CMG foi mais pronunciada entre mulheres com mais de 35 anos de idade e entre mulheres com diagnóstico de CMG 12 meses após o parto (Cieto et al., 2021). Foi relatado que mulheres com estágios mais avançados da doença durante a gravidez têm uma sobrevida reduzida quando comparadas ao grupo controle (Monteiro et al., 2019). Nos casos acompanhados neste estudo as pacientes foram a óbito em uma faixa de 5 meses a 18 meses após o diagnóstico. Já em Cieto et al. (2021) observou-se que 42\% das pacientes com CMG foram a óbito. 


\section{Considerações Finais}

O CMG é considerado um evento desafiador e raro, apesar da incidência, com o retardo da gravidez para após os 30 anos, estar aumentando. Exige um delineamento terapêutico individualizado considerando o estadiamento da doença, idade gestacional e o equilíbrio entre os riscos e benefícios para a mãe e para o feto. Neste estudo as principais RAM apresentadas pelas pacientes foram leves, principalmente gastrointestinais e bioquímicas. A possibilidade de contribuição do farmacêutico na identificação precoce das RAM e no auxílio do seu manejo pelo paciente reforçam a potencialidade da inclusão desse profissional na equipe multiprofissional que deve acompanhar este tipo de paciente. Tal equipe deverá ter sua ação centrada no bem-estar materno-fetal, contando com oncologista clínico, ginecologista obstetra, farmacêutico, nutricionista, psicólogo e assistente social para atender a paciente e o feto de forma integral. Desta forma, os resultados apresentados nesse estudo demonstram a importância do desenvolvimento de novos artigos sobre o impacto do tratamento oncológico em pacientes grávidas, estudos com uma população maior e um grupo controle são importantes para elucidar comparações a respeito de RAM e sobrevida entre pacientes com câncer de mama não gestantes e gestantes.

\section{Referências}

Avery, M. \& Williams, F. (2015). The importance of pharmacist providing patient education in oncology. Journal of Oncology Pharmacy Practice, 28(1): 2630. http://journals.sagepub.com/doi/10.1177/0897190014562382

Bae, S. Y., Kim, K. S., Kim, J. S., Lee, S. B., Park, B., Lee, S. W., Lee, H. J., Kim, H. K., You, J., \& Jung, S. P. (2018). Neoadjuvant Chemotherapy and Prognosis of Pregnancy-Associated Breast Cancer: A Time-Trends Study of the Korean Breast Cancer Registry Database. Journal of Breast Cancer, 21(4): 425-432.https://doi.org/10.4048/jbc.2018.21.e58

Bell, R. J., Fradkin, P., Parathithasan, N., Robinson, P. J., Schwarz, M., \& Davis, S. R. (2013). Pregnancy-associated breast cancer and pregnancy following treatment for breast cancer, in a cohort of women from Victoria, Australia, with a first diagnosis of invasive breast cancer. Breast, 22(5):980985.https://doi.org/10.1016/j.breast.2013.05.013

Bezerra, A. L. R., Bezerra, M. C. M., Santos, A. F. F. L., Lima, F. M., Almeida, I. T. B., \& Ferreira, L. M. G. (2019). Evaluation of delays in diagnosis and treatment of breast cancer in a reference center: a retrospective analysis Mastology, 29(3): 144-51. http://www.mastology.org/wpcontent/uploads/2019/10/MAS-v29n3_144-151.pdf

Biganzoli, L., Aapro, M., Loibl, S., Wildiers, H., \& Brain, E. (2016). Taxanes in the treatment of breast cancer: Have we better defined their role in older patients? A position paper from a SIOG Task Force. Cancer Treatment Reviews, 43: 19-26. https://doi.org/10.1016/j.ctrv.2015.11.009

Case, A. S. (2016). Pregnancy-associated Breast Cancer. Clinical Obstetrics and Gynecology, 59(4): 779-788. http://doi.org/10.1097/GRF.0000000000000235

Castro, I. J., Busom, M. P., Cabañas, G. I. B., Montero, E. R., Méndez, A. A., Chivato, R. C., \& Ruiz, M. C. (2013). Estudio de las reacciones adversas relacionadas con la infusión de paclitaxel y docetaxel. Farmacia Hospitalaria, 37(2):88-94. http://doi.org/10.7399/FH.2013.37.2.374

Chiu, N., Chiu, L., Chow, R., Lam, H., Verma, S., Pasetka, M., Chow, E., \& DeAngelis, C. (2017). Taxane-induced arthralgia and myalgia: A literature review. Journal of Oncology Pharmacy Practice, 23(1): 56-67. http://journals.sagepub.com/doi/10.1177/1078155215627502

Cieto, J. F., Santos, L. A. C., \& Gozzo, T. O. (2021). Câncer durante a gravidez: análise dos casos com ênfase nos resultados obstétricos e neonatais. Revista de Enfermagem do Centro-Oeste Mineiro, 4096 (11): 1-10.http://doi.org/10.19175/recom.v10i0.4096

Cipriano, P., \& Oliveira, C. (2016). Gestação e câncer de mama: proposta de guia de orientações. Fisioterapia Brasil, 17(2):14857.https://doi.org/10.33233/fb.v17i2.202

Cordeiro, C. N., \& Gemignani, M. L. (2017). Breast Cancer in Pregnancy: Avoiding Fetal Harm When Maternal Treatment Is Necessary. The Breast Journal, 23(2): 200-205.https://doi.org/10.1111/tbj.12780

Ferlay, J., Colombet, M., Soerjomataram, I., Mathers, C., Parkin, D. M., Piñeros, M., Znaor, A., \& Bray, F. (2018). Estimating the global cancer incidence and mortality in 2018: GLOBOCAN sources and methods. International Journal of Cancer, 68 (6): 394-424. https://onlinelibrary.wiley.com/doi/abs/10.1002/ijc.31937

Kettelhut, J. C., \& Modena, M. A. B. (2008). Câncer de mama e gestação. Revista Faculdade de Ciências Médicas Sorocaba, 10(4): 1-4. https://revistas.pucsp.br/index.php/RFCMS/article/view/985/984

Merviel, P., Jouvance, O., Naepels, P., Fauvet, R., Cabry-Goubret, R., Gagneur, O., \& Gondry, J. (2011). Existe-t-il encore desfacteurs de risqué de survenue

d'um câncer dusein? Gynécologie Obstétrique $\quad$ \& $\quad$ Fertilité,

https://10.1016/j.gyobfe.2010.10.015

Ministério da Saúde. Instituto Nacional de Câncer José Alencar Gomes da Silva. Estimativa 2020: Incidência de Câncer no Brasil. Rio de Janeiro; 2020. https://www.inca.gov.br/sites/ufu.sti.inca.local/files/media/document/estimativa-2020-incidencia-de-cancer-no-brasil.pdf 
Research, Society and Development, v. 10, n. 16, e559101624129, 2021

(CC BY 4.0) | ISSN 2525-3409 | DOI: http://dx.doi.org/10.33448/rsd-v10i16.24129

Monteiro, D. L. M., Nunes, C. L., Rodrigues, N. C. P, Antunes, C. A., Almeida, E. M., Barmpas, D. B. S., Magalhães, A. L. C., \& Trajano, A. J. B. (2019) Fatores associados ao câncer de mama gestacional: estudo caso-controle. Ciência \& Saúde Coletiva, 24(6):2361-2369.https://doi.org/10.1590/141381232018245.18392017

Prado, N., Loiola, P., Guimarães, T., Ohara, E. C. C., \& Oliveira, L. D. R. (2020). Gestante com diagnóstico de câncer de mama: prevenção, diagnóstico e assistência. Brazilian Journal of Health Review, 3(1): 1109-1131. https://doi.org/10.34119/bjhrv3n1-086

Pereira, A. S., Shitsuka, D. M., Parreira, F. J., \& Shitsuka, R. (2018). Metodologia da pesquisa científica. UFSM.

Silva, K. M., Rockenbach, B. F., Moura, J. E., \& Souza, A. B. A. (2018). Câncer de mama na gestação: abordagem diagnóstica e terapêutica. Acta medica, 39(2): 61-69. https://pesquisa.bvsalud.org/portal/resource/pt/biblio-987625

Souza, C. B., Fustinoni, S. M., \& Amorim, M. H. C. (2015). Estudo do tempo entre o diagnóstico e início do tratamento do câncer de mama em idosas de um hospital de referência em São Paulo, Brasil. Ciência saúde coletiva, 20(12): 3805-16. http://www.scielo.br/scielo.php?script=sci_arttext\&pid=S1413$81232015001203805 \& \operatorname{lng}=\mathrm{pt} \& \operatorname{lng}=\mathrm{pt}$

Suzuki, H., Suzuki, S., Kamata, H., Sugama, Y., Demachi, K., Kiwako, I., Igarashi, T., \& Yamaguchi, M. (2019). Impact of pharmacy collaborating services in an outpatient clinic on improving adverse drug reactions in outpatient cancer chemotherapy Journal of Oncology Pharmacy Practice, 25(7): 1558-63. http://journals.sagepub.com/doi/10.1177/1078155218798138

Walker, L. G., Eremin, J. M., Aloysius, M. M., Vassanasiri, W., Walker, M. B., El-Sheemy, M., Cowley, G., Beer, J., Samphao, S., Wiseman, J., Jibril, J. A., Valerio, D., Clarke, D. J., Kamal, M., Thorpe, G. W., Baria, K., \& Eremin, O. (2011). Effects on quality of life, anti-cancer responses, breast conserving surgery and survival with neoadjuvant docetaxel: a randomised study of sequential weekly versus three-weekly docetaxel following neoadjuvant doxorubicin and cyclophosphamide in women with primary breast cancer. BMC Cancer, 179 (11): 1-11. http://bmccancer.biomedcentral.com/articles/10.1186/1471-240711-179 